\title{
The importance of ultrasonographic pneumatosis intestinalis in equine neonatal gastrointestinal disease
}

\author{
C. NAVAS de SOLIS*, J. E. PALMER, R. C. BOSTON and V. B. REEF \\ New Bolton Center, University of Pennsylvania, Kennett Square, Pennsylvania, USA \\ *Correspondence email: navasdes@vet.upenn.edu; Received: 01.03.11; Accepted: 08.06.11
}

\section{Summary}

Reasons for performing study: Recognising the presence of a necrotising component of the gastrointestinal disease may be clinically useful in ill equine neonates.

Objectives: To study the importance of abdominal sonograms in neonatal foals suffering from gastrointestinal conditions and to describe the clinical features of necrotising gastrointestinal disease.

Hypothesis: There is a subgroup of neonates with sonographically detectable pneumatosis intestinalis (PI), reflecting a necrotising disease.

Methods: Records of foals aged $\leq 7$ days hospitalised from 2005 to 2009 with signs of gastrointestinal disease were evaluated $(n=89)$. The association of sonographic, clinical and clinicopathological signs with necrotising gastrointestinal disease and outcome was determined.

Results: PI was imaged in 19 foals. Twenty-seven foals were classified as having necrotising gastrointestinal disease based on the presence of gastrointestinal signs (colic, diarrhoea, gastric reflux or abdominal distension) and sonographic PI ( $n=19)$, surgical $(n=2)$ or pathological $(n=6)$ evidence of gastrointestinal necrosis. There was a difference between survival rate in foals with and without necrotising disease $(33.3$ and $69.4 \%$, respectively, $\mathrm{P}=0.005)$ or foals with and without PI detected sonographically (36.8 and $72.1 \%$, respectively, $P=0.023$ ). PI was the only sonographic finding associated with outcome. Prematurity, the presence of blood in the faeces, gastric reflux, abdominal distension, abnormal echogenicity of the colon and the lowest white blood cell count during hospitalisation were associated with necrotising gastrointestinal disease $(P<0.05)$.

Conclusions and potential relevance: Abdominal sonograms have prognostic value in neonatal gastrointestinal disease. PI and the presence of necrotising gastrointestinal disease were common and associated with a poor prognosis.

Keywords: horse; pneumatosis intestinalis; necrotising gastrointestinal disease; neonate; abdominal ultrasonography

\section{Introduction}

Gastrointestinal (GI) disease is common in equine neonates. Foals presented for abdominal pain or diarrhoea may be easily identified as suffering from GI disease. However, neonates with other clinical presentations, such as sepsis, prematurity or perinatal asphyxia syndrome, may show nonspecific signs, with $\mathrm{Gl}$ disease being one of many components of the process. Furthermore, clinical and clinicopathological signs overlap between these other disease conditions. Case management varies between various diseases and prognoses differ. Abdominal ultrasonography has proved useful in the assessment of human and veterinary cases with different conditions. In human critical care units, the time from presentation to operative care, length of hospitalisation, complications and cost decrease when abdominal sonograms are performed during emergency evaluations, potentially due to a more rapid diagnosis (Levy and Noble 2008). In paediatric colitis (Baud et al. 2004) and Gl emergencies (Puylaert 2001; Pastore et al. 2007) sonographic findings correlate with anatomical distribution of the disease or diagnosis and often help patient management. Abdominal ultrasonography is well described in equine neonates (Reef 1998; McAuliffe 2004; Reef 2004; Porter and Ramirez 2005); however, studies that quantify its us efulness, accuracy and limitations are scarce.

Necrotising enterocolitis (NEC) is a disease process of the GI tract of neonates that results in inflammation and bacterial invasion of the bowel wall. Necrotising enterocolitis occurs frequently in human newborn intensive care units (Babcock 2002; Thompson and Bizzarro 2008), primarily in premature neonates (Lee and Polin 2003; Obladen 2009). Necrotising enterocolitis has been described in foals in isolated case reports (Cudd and Pauly 1987; Yager 1987; Jones et al. 1988; East et al. 1998) and several texts have suggested that it may be an under recognised syndrome (Slovis 2003; Magdesian 2005; Vaala 2009; Wilkins 2010). The diagnosis of NEC in human infants is based on clinical and imaging findings. The presence of intramural gas (pneumatosis intestinalis, $\mathrm{Pl}$ ) is considered virtually pathognomonic for necrotising enterocolitis in neonates with a compatible clinical picture (Walsh and Kliegman 1986; St. Peter et al. 2003; Epelman et al. 2007; Kim et al. 2007). Radiographic documentation of PI is the current standard to confirm NEC diagnosis in man, but the sonographic detection of PI has been shown to be both sensitive and specific (Kim et al.
2007) and to have a growing and evolving role in the evaluation of these patients (Epelman et al. 2007).

This retrospective study was designed to describe the abnormalities present in abdominal sonograms of equine neonates with signs of $\mathrm{Gl}$ disease and the clinical and clinicopathological findings in neonates with necrotising $\mathrm{Gl}$ disease. The data were analysed to study the association of sonographic, clinical and clinicopathological abnormalities with the presence of sonographic PI, necrotising $\mathrm{Gl}$ disease and outcome. We hypothesised that the sonographic signs in foals with GI disease are varied but there is a subgroup of neonates with characteristic sonographic findings (including PI) and a clinical presentation suggestive of a necrotising component of the $\mathrm{Gl}$ disease. We also hypothesised that foals with necrotising GI disease and PI have a poor prognosis.

\section{Materials and methods}

\section{Case selection}

Records of foals aged $\leq 7$ days presented between 31 December 2005 and 31 December 2009 with clinical signs of GI disease (colic, diarrhoea, gastric reflux or abdominal distension) or that developed these signs during hospitalisation were studied retrospectively. Clinical, clinicopathological and sonographic data were obtained from medical records. Videotapes of the sonographic examinations were reviewed blindly by one of the authors (C.N.S.).

\section{Ultrasonographic interpretation}

Categorical sonographic variables included in the analysis were: abdominal sonogram performed during hospitalisation; presence and location of $\mathrm{PI}$ within the Gl tract; presence of abnormalities in echogenicity of the stomach, duodenum, jejunum, ileum, colon and caecum; presence of ileus within the Gl tract; presence of abnormal stomach contents; presence of functional abnormalities in the duodenum, jejunum, ileum, colon or caecum, presence of meconium; presence and distribution of abnormal peritoneal fluid; and presence of abnormalities in the liver. PI was identified 


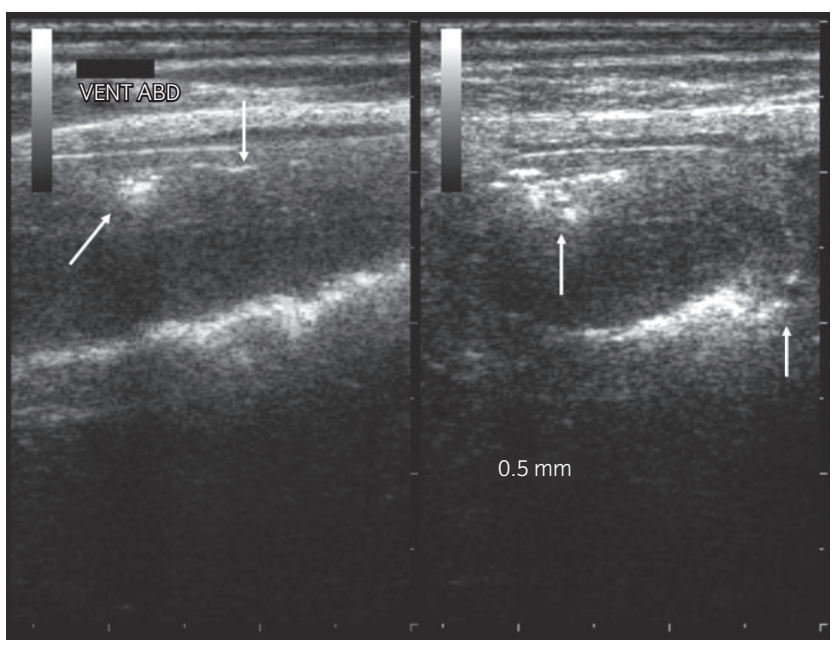

Fig 1: Sonogram of the ventral abdomen obtained from a foal with enterocolitis. Notice the hyperechoic intramural gas echoes (arrows) within a loop of thickened small intestine $(5 \mathrm{~mm})$. The gas echoes are imaged in mutually perpendicular longitudinal (left) and transverse (right) planes.

when hyperechoic echoes casting a dirty shadow were imaged within the bowel wall consistent with intramural gas (Fig 1 and Supporting information). Abnormalities in bowel wall echogenicity were areas of increased, decreased or heterogeneous echogenicity within the intestinal wall (Fig 2). Functional abnormalities of the gastrointestinal tract were defined as abnormalities in the contents or motility of the affected portion of the Gl tract. Motility was considered normal if there were frequent, rhythmic peristaltic waves. Subjectively increased, decreased or arrhythmic contractions were considered abnormal. The stomach was not evaluated for motility disorders. Ileus was defined as diffuse hypomotility and distension of the gastrointestinal viscera (Fig 3). Abnormal peritoneal fluid was defined as fluid that is hypoechoic to echoic or increased in amount (Reef 2004; Porter and Ramirez 2005) (Fig 4). The presence of focally abnormal peritoneal fluid (vs. diffusely) was analysed as a separate variable.

\section{Data recorded}

Categorical clinical and clinicopathological variables obtained from records were outcome, presenting complaint, prematurity, feeding

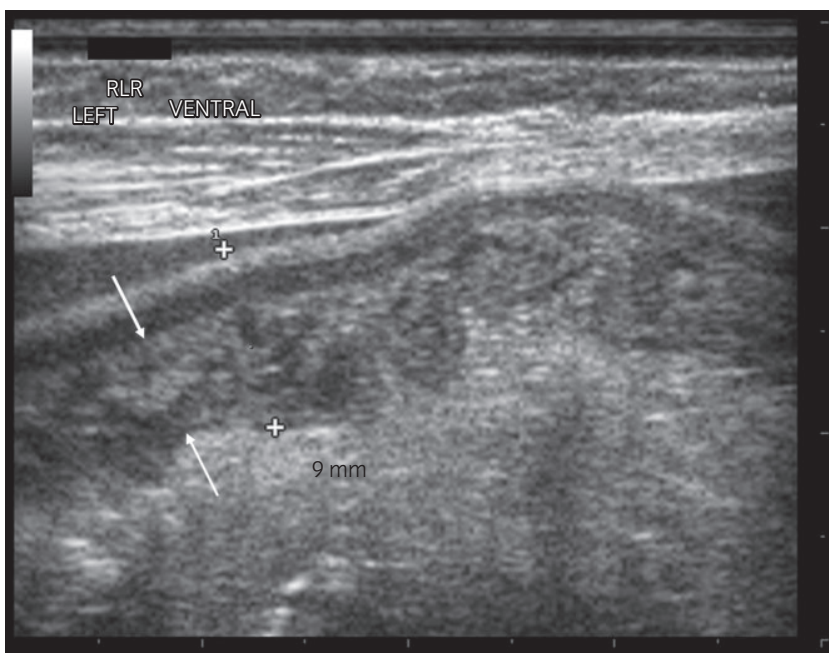

Fig 2: Sonogram of the large intestine obtained from a foal with enterocolitis. Notice the increased thickness of the wall $(9 \mathrm{~mm})$ and the thickened heteroechoic submucosa (arrow).

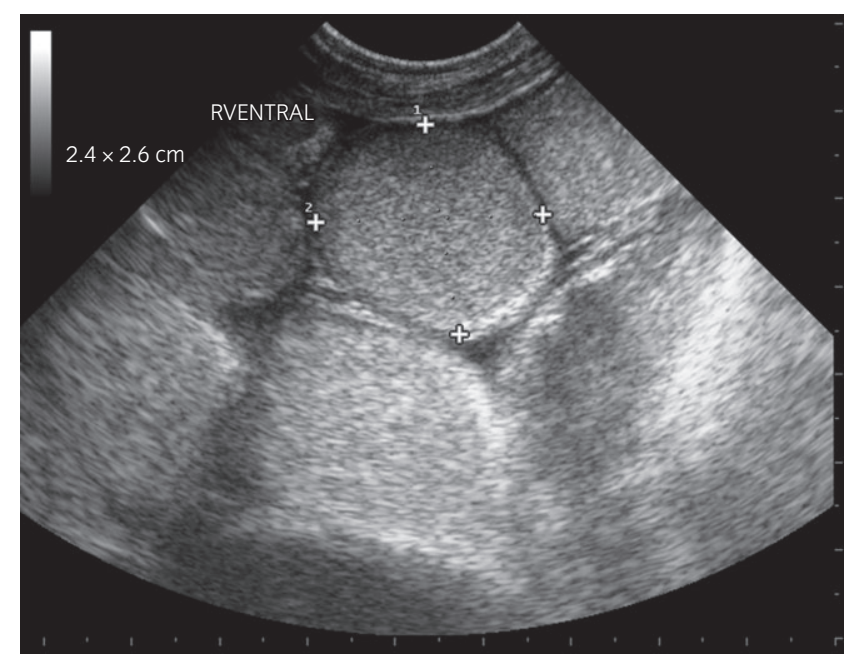

Fig 3: Sonogram of the abdomen obtained from a foal with enterocolitis. Notice the distended loops of small intestine consistent with ileus.

strategy, presence of necrotising gastrointestinal disease, presence of colic, diarrhoea, gastric reflux, blood in faeces, abdominal distension, pneumonia, respiratory distress, bacteraemia, clostridiosis, salmonellosis and congenital heart disease. The presenting complaint was categorised as colic, diarrhoea or other. If there were multiple complaints, the problem prompting hospitalisation was chosen for analysis. Foals were considered premature or to have respiratory distress if clinicians recorded these in the medical records. Foals were considered to have pneumonia if recorded by the clinician or pathologist. Necrotising gastrointestinal disease was defined as signs of GI disease and sonographic evidence of PI or surgical or pathological evidence of necrosis of the intestinal wall.

Continuous sonographic variables recorded were the thickness of stomach, duodenum, jejunum, ileum, colon and caecum. The continuous clinicopathological variables recorded were highest white blood cell count (WBCC), lowest WBCC, lowest total protein (TP), highest fibrinogen, plasma IgG concentration (DVM Rapid Test) ${ }^{a}$ at presentation, highest lactate concentration, lowest $\mathrm{PaO}_{2}$ and highest $\mathrm{PaCO}_{2}$.

\section{Data analysis}

For the statistical analysis, the uniqueness of each record was checked. Fisher's exact test was used to detect the association between pairs of

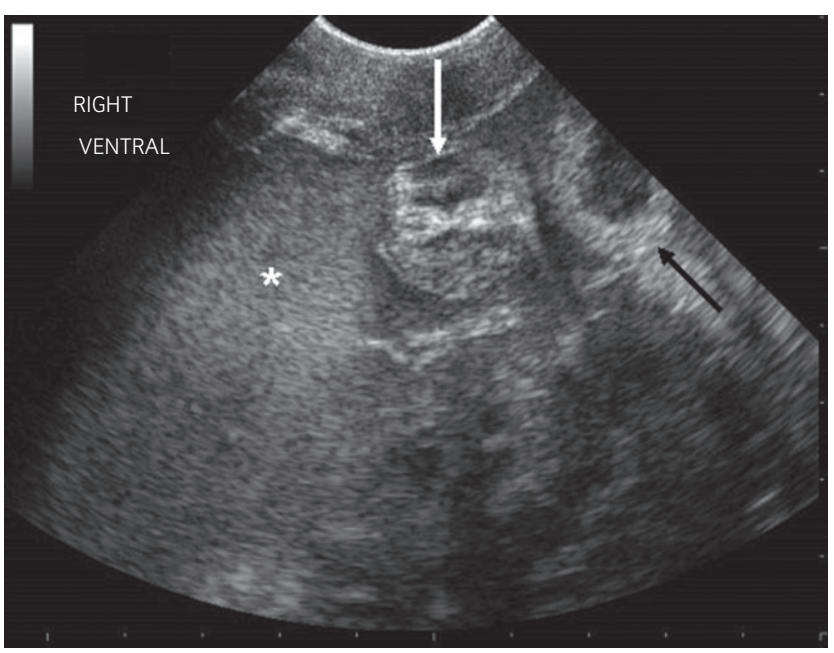

Fig 4: Sonogram of the ventral abdomen of a foal with enterocolitis. Notice the increased amount of echoic peritoneal fluid (asterisk), surrounding loops of ileum (white arrow) and jejunum (black arrow). 
categorical variables and to assess the significance of such associations. Logistic regression was used to quantify the associations between dichotomous outcomes and plausible metabolic drivers. For polychotomous outcomes, associations were detected by the Kruskall-Wallis procedure and the strength, sense and significance of the associations additionally confirmed with the aid of Somer's D procedure. Differences were reported as observed. The statistical software Stata $11.1^{\mathrm{b}}$ was used for the analysis. A P value of 0.05 was used to distinguish significant from nonsignificant associations. Only foreshadowed associations were explored, in keeping with the study design.

\section{Results}

The inclusion criteria were metby 115 foals. Twenty-sixfoals were eliminated because of insufficient information in the medical records; therefore data from 89 foals were analysed. Variables related to the sonographic appearance of the ileum were excluded from the analysis owing to insufficient data. Variables related to feeding strategies were excluded owing to heterogeneity of feedings before presentation and changes during hospitalisation. There were 48 males and 41 females. There were 58 Thoroughbreds, 15 Standardbreds, 6 Warmbloods, 4 Arabians and 6 foals of other breeds. Twenty-four foals presented for colic, 8 for diarrhoea (4 had colic and diarrhoea as presenting complaints but abdominal pain prompted referral) and 57 presented with other complaints. These included weakness/depression ( $n=15)$, the result of a high risk pregnancy $(n=7)$, dystocia $(n=6)$, neonatal encephalopathy $(n=6)$, recumbency $(n=4)$, straining to urinate $(n=4)$, failure to nurse $(n=3)$, premature placental separation $(n=3)$, respiratory distress $(n=2)$, prematurity $(n=2)$, ruptured bladder $(n=1)$, septic shock $(n=1)$, anaemia $(n=1)$, flexural deformity $(n=1)$ and haemorrhage $(n=1)$. Forty-seven neonates did not have signs of gastrointestinal disease recorded at presentation and developed them during hospitalisation. The mean \pm s.d. age at presentation was $1.9 \pm 1.4$ days. Abdominal ultrasound examinations were performed stall-side in 62 neonates. PI was most frequently imaged as scattered hyperechoic foci within the intestinal wall, although large clusters of hyperechoic foci within the bowel wall were also imaged in some foals. The intramural gas was imaged more frequently in the small intestine $(57.9 \%, 11 / 19)$ than in the large intestine $(31.6 \%, 6 / 19)$, and infrequently $(10.5 \%, 2 / 19)$ in both the small and the large intestine. Twenty-seven foals were classified as having necrotising disease based on the clinical signs of GI disease and sonographic evidence of $\mathrm{PI}$ (19), and/or surgical (2) or pathological (6) evidence of necrosis. Post mortem examinations were performed in 4 foals with sonographic $\mathrm{Pl}$ and, in 2 cases, histology was consistent with necrotising disease. Another foal with sonographic PI had an exploratory laparotomy and showed extensive macroscopic abnormalities most consistent with necrotising GI disease, including intramural gas.

Summaries of the clinical/clinicopathological and sonographic abnormalities of all foals and foals having necrotising GI disease are shown in Tables 1 and 2, respectively. Exploration of associations of clinical and clinicopathological abnormalities with the presence of $\mathrm{PI}$ revealed that the lowest WBCC $(P=0.029)$ and lowest TP $(P=0.032)$ were associated with the presence of PI. Exploration of associations of sonographic, clinical and clinicopathological abnormalities with the presence of necrotising GI disease and outcome revealed that PI was the only sonographic change associated with survival $(P=0.023)$. The survival rate was $36.8 \%(7 / 19)$ if $\mathrm{PI}$ was imaged sonographically vs. $72.1 \%$ (31/43) in foals that had abdominal scans and $\mathrm{Pl}$ was not imaged. There was also an association between outcome and necrotising $\mathrm{Gl}$ disease $(P=0.005)$. Only $33.3 \%(9 / 27)$ of foals with necrotising disease survived vs. $69.4 \%$ (43/62) of foals without evidence of necrotising disease. The presence of necrotising GI disease was associated with prematurity $(P=0.03$, odds ratio [OR] 5.1), the presence of blood in the faeces $(P=0.009)$, gastric reflux $(P=0.003)$, abdominal distension $(P=0.009)$ and the lowest WBCC $(P<0.001)$. Detection of abnormal echogenicity of the colon was associated with the presence of necrotising disease $(P=0.015)$.

Clinical or clinicopathological variables associated with outcome were presence of pneumonia $(P=0.01)$, respiratory distress $(P=0.008)$, lactate concentration $(P<0.001)$, highest WBCC $(P=0.034)$, lowest WBCC $(P=0.025)$, highest fibrinogen $(P=0.02)$, and highest $\mathrm{PaCO}_{2}(P=0.014)$.
TABLE 1: Clinical and clinicopathological variables in foals with GI disease

\begin{tabular}{|c|c|c|}
\hline & All cases & $\begin{array}{l}\text { Cases with } \\
\text { necrotising Gl }\end{array}$ \\
\hline Complaint & $\begin{array}{l}24 \text { colic, } 8 \text { diarrhoea } \\
\text { and } 57 \text { other }\end{array}$ & $\begin{array}{l}7 \text { colic, } 3 \text { diarrhoea, } \\
17 \text { other }\end{array}$ \\
\hline Prematurity & $13 / 46(28.2 \%)$ & $7 / 13(53.8 \%)^{*}$ \\
\hline Survived to discharge & $52 / 89(58.4 \%)$ & $9 / 27(33.3 \%)^{*}$ \\
\hline Colic & $59 / 81(72.8 \%)$ & $20 / 24(83.3 \%)$ \\
\hline Reflux & $20 / 80(25 \%)$ & $11 / 23(47.8 \%)^{*}$ \\
\hline Abdominal distension & $52 / 78(66.6 \%)$ & $19 / 21(90.4 \%)^{*}$ \\
\hline Diarrhoea & $27 / 78(34.6 \%)$ & $10 / 23(43.5 \%)$ \\
\hline Blood in faeces & $12 / 79(15.2 \%)$ & $8 / 23(34.8 \%)^{*}$ \\
\hline Congenital heart disease & $3 / 83(3.6 \%)$ & $2 / 26(7.7 \%)$ \\
\hline Pneumonia & $22 / 86(25.6 \%)$ & $9 / 26(34.6 \%)$ \\
\hline Respiratory distress & $17 / 85(20 \%)$ & $4 / 25(16 \%)$ \\
\hline $\mathrm{TP}(g / l)$ & $51 \pm 11$ & $50 \pm 15$ \\
\hline Lactate $(\mathrm{mmol} / \mathrm{l})$ & $6.7 \pm 4.9$ & $6.7 \pm 4.7$ \\
\hline Fibrinogen $(g / l)$ & $5.73 \pm 2.00$ & $5.53 \pm 2.57$ \\
\hline IgG & $688.3 \pm 654.4$ & $619.3 \pm 646.5$ \\
\hline Surgery & $7 / 89$ (7.9\%) & $2 / 27(7.4 \%)$ \\
\hline Highest WBCC $\left(10^{9}\right.$ cells/l) & $8.73 \pm 4.66$ & $7.35 \pm 4.47$ \\
\hline Lowest WBCC (109 cells/l) & $4.84 \pm 3.29$ & $3.76 \pm 3.14^{*}$ \\
\hline Clostridium toxins & $8 / 31 \quad(25.8 \%)$ & $3 / 16(18.7 \%)$ \\
\hline Salmonella PCR & $7 / 47(14.9 \%)$ & $2 / 14(14.3 \%)$ \\
\hline Bacteraemia & $32 / 66$ (48.5\%) & $12 / 22(54.6 \%)$ \\
\hline Lowest $\mathrm{PaO}_{2}$ (mmHg) & $59.0 \pm 18.6$ & $62.3 \pm 17.4$ \\
\hline Highest $\mathrm{PaCO}_{2}(\mathrm{mmHg})$ & $57.4 \pm 24.3$ & $56.5 \pm 16.8$ \\
\hline
\end{tabular}

Results are presented as abnormal individuals/total and percentages in parentheses. Continuous data are presented as mean \pm s.d. TP $=$ total protein. FPT $=$ failure of passive transfer. $\mathrm{WBCC}=$ white blood cell count. * denotes variables for which significant differences were found between the 2 groups $(\mathrm{P}<0.05)$.

Prematurity was a perfect predictor of outcome $(0 / 13$ premature foals survived to discharge).

\section{Discussion}

Abnormalities in all parts of the GI tract and abdominal viscera were found in the sonograms of neonates with signs of Gl disease, supporting the importance of performing comprehensive abdominal ultrasound examinations in this group. Using high-frequency ultrasound transducers, the thickness, echogenicity and layering of the wall of the GI viscera can be evaluated (Porter and Ramirez 2005). Different patterns of abnormal intestinal echogenicity, based on the loss/maintenance of stratification and folding, have been shown to help define the GI disease process in children with colitis (Baud et al. 2004). In this series of cases abnormalities in the Gl wall, echogenicity was frequently associated with the diagnosis of necrotising Gl disease. However, we did not analyse changes in wall layering and the lack of definitive diagnosis in many cases would have made pathological correlations difficult. Further studies are needed to investigate the association of different sonographic patterns with specific diagnoses in foals.

The radiographic diagnosis of $\mathrm{PI}$ had been reported in 2 equine neonates (Cudd and Pauly 1987). To our knowledge, this is the first report of the sonographic detection of $\mathrm{PI}$ in foals and evaluation of its prognostic value. The sonographic appearance of PI in the equine neonates was most frequently that of scattered hyperechoic foci, although clustered foci were imaged in some instances. Detailed images using high frequency transducers allow for the differentiation between intraluminal and intramural gas, for detection of small amounts of intramural gas and for determining the extent of the PI. The pattern or extent of PI does not correlate with the severity of the symptoms in clinical NEC in human neonates (St. Peter et al. 2003), but it did in an experimental model in 
TABLE 2: Sonographic findings in foals with gastrointestinal disease

\begin{tabular}{|c|c|c|}
\hline & All cases & $\begin{array}{l}\text { Cases with } \\
\text { necrotising Gl }\end{array}$ \\
\hline Sonogram performed & $62 / 89(69.9 \%)$ & $24 / 27$ (88.9\%) \\
\hline Abnormal region of GI & $\begin{array}{l}\text { SI 19/52, LI 8/52, } \\
\text { none 4/52, } \\
\text { SI and LI 20/52 }\end{array}$ & $\begin{array}{c}\text { SI 8/23, LI 5/23, } \\
\text { none 0/23, } \\
\text { SI and LI 10/23 }\end{array}$ \\
\hline Ileus & $15 / 57$ (26.3\%) & $7 / 23$ (30.4\%) \\
\hline $\mathrm{PI}$ & $19 / 62(30.6 \%)$ & $19 / 24(79.1 \%)^{*}$ \\
\hline Gastric echogenicity & $1 / 45(2.2 \%)$ & $1 / 21 \quad(4.7 \%)$ \\
\hline Gastric contents & $8 / 41 \quad(19.5 \%)$ & $5 / 19(26.3 \%)$ \\
\hline Gastric wall thickness (mm) & $3.4 \pm 2.1$ & $3.4 \pm 2.8$ \\
\hline Duodenal echogenicity & $1 / 24$ (4.2\%) & $0 / 10(0 \%)$ \\
\hline Duodenal function & $19 / 33(57.6 \%)$ & $11 / 17(64.7 \%)$ \\
\hline Duodenal wall thickness (mm) & $2.3 \pm 1.1$ & $2.4 \pm 1.0$ \\
\hline Jejunal echogenicity & $17 / 54(31.4 \%)$ & $12 / 22(54.5 \%)$ \\
\hline Jejunal function & $36 / 54(66.7 \%)$ & $15 / 23(65.2 \%)$ \\
\hline Jejunal wall thickness (mm) & $3.3 \pm 1.7$ & $3.5 \pm 2.0$ \\
\hline Colonic echogenicity & $10 / 56(17.8 \%)$ & $10 / 22(45.4 \%)^{*}$ \\
\hline Colonic function & $25 / 52(48.1 \%)$ & $12 / 22$ (54.5\%) \\
\hline Colonic wall thickness (mm) & $2.6 \pm 1.3$ & $3.1 \pm 1.6$ \\
\hline Caecal echogenicity & $3 / 21 \quad(14.2 . \%)$ & $2 / 7$ (28.5\%) \\
\hline Caecal function & $8 / 14$ (57.1\%) & $6 / 7(85.7 \%)$ \\
\hline Caecal wall thickness (mm) & $2.5 \pm 1.8$ & $2.5 \pm 2.3$ \\
\hline Peritoneal fluid & $14 / 54(25.9 \%)$ & $5 / 20(25.0 \%)$ \\
\hline Focal fluid & $1 / 54(1.8 \%)$ & $1 / 20(5 \%)$ \\
\hline Liver & $1 / 57(1.8 \%)$ & $0 / 22(0 \%)$ \\
\hline
\end{tabular}

Results are presented as abnormal individuals/total and percentages in parenthesis. Continuous data are presented as mean \pm s.d. $\mathrm{PI}=$ pneumatosis intestinalis. $\mathrm{SI}=$ small intestine, $\mathrm{LI}=$ large intestine. * denotes variables for which significant differences were found between the 2 groups $(P<0.05)$.

rabbits (Kim et al. 2007). We did not attempt to describe a pattern of PI nor to quantify or grade the severity of the PI in our foals because of the retrospective nature of the study. Intramural gas may involve any part of the GI tract in human NEC but is more commonly present in the distal small bowel and large bowel and imaged more commonly in the right lower quadrant (Kim et al. 2007). In our study, the intramural gas was imaged more frequently in the small intestine than in the large intestine and was detected infrequently in both small and large intestine.

Sonograms were performed stall-side without the need for anaesthesia. Other imaging modalities may be more difficult to perform in the equine neonatal intensive care unit setting. With the exception of occasional radiographs, other imaging modalities were not used in this group of neonates and we did not attempt comparison with other potentially useful imaging techniques (radiography, computed tomography or magnetic resonance imaging).

Of the many sonographic features of NEC, a recent study in human neonates showed that an adverse outcome was associated with the presence of free peritoneal gas, focal fluid collections or 3 or more of the following: increased bowel wall echogenicity, absent bowel perfusion, portal venous gas, bowel wall thinning, bowel wall thickening, free peritoneal fluid with echoes, and intramural gas (Silva et al. 2007). Sonographic assessment of intestinal vascularisation was not routinely performed in the foals in this study and thus bowel perfusion could not be critically evaluated. Further studies are needed to characterise intestinal vascularisation using colour flow Doppler ultrasound in equine species and to determine its value as a diagnostic tool.

In our analysis, PI was the only sonographic finding associated with a poor outcome. Pneumatosis intestinalis is not a diagnosis or a clinical problem per se, but a pathological or imaging finding that is a consequence of an underlying disease (St. Peter et al. 2003). PI has been attributed to at least 58 causes in man (King and Suckett 1992; Gurland et al. 1998; Silva et al. 2007) although it is virtually pathognomonic of NEC in human neonates with compatible clinical presentation (Kim et al. 2007). The imaging findings can therefore only be interpreted in the context of the neonatal patient with an appropriate clinical presentation.

The precise aetiology of NEC is unknown, but its aetiopathology probably represents an interaction of factors leading to intestinal inflammation and bacterial invasion. Wall ischaemia is the final pathway and not the primary initiator of the PI (Lee and Polin 2003; Thompson and Bizzarro 2008). Prematurity is the only independent determinant of human NEC (Lee and Polin 2003), and prematurity and dystocia (Cudd and Pauly 1987) have been suggested to be associated with NEC in foals. In this retrospective study, there was a difference $(P=0.03, O R 5.1)$ in the presence of NEC between premature $(7 / 13,53.8 \%)$ and term $(6 / 3218.75 \%)$ foals. However, the actual breeding date was seldom recorded in the medical record, and also conclusions should be drawn with caution. Even though in this group premature foals were more likely to develop necrotising disease (5.1 times more likely as determined by the OR), foals analogous to premature very low birthweight children are not frequent. Therefore, direct extrapolation of information from human NEC to necrotising $\mathrm{Gl}$ disease in equine neonates may not yield applicable conclusions in all instances.

Three factors are felt to be necessary for NEC to occur in human neonates: ischaemic/hypoxic mucosal injury, feeding and the presence of bacteria (Lee and Polin 2003). These factors may be present in critically ill equine neonates. Respiratory distress syndrome or congenital heart disease has been suggested to play a role in the pathogenesis of NEC in human neonates by causing hypoxic mucosal injury (Guner et al. 2008). We tested the association between respiratory disease, arterial blood gases values and congenital heart disease with the presence of necrotising gastrointestinal disease and no association was found. The number of neonates with congenital heart disease in this series was small $(n=3)$ and therefore the lack of association could be due to lack of statistical power. Bacteraemia has been documented in up to $35 \%$ of NEC cases in children (Lee and Polin 2003) and some authors have hypothesised that NEC is a contagious disease, implicating Klebsiella, E. coli, Enterobacter, Salmonella, Pseudomona, Clostridia, Staphylococci, coronavirus, rotavirus or enterovirus (Walsh and Kliegman 1986). There are reports associating C. difficile (Jones et al. 1988), C. perfringens (East et al. 1998) and Rhodococcus equi (Yager 1987) with NEC in foals. Blood cultures, polymerase chain reactions for detection of salmonella in faeces and faecal testing for the detection of clostridial toxins were performed frequently in the foals reported herein, and a positive result was not associated with a necrotising component to their Gl disease.

Progression of NEC in human neonates may take a variable period, although it can be an extremely rapid process. Sonographic findings match the pathological stages and may precede clinical signs (Faingold et al. 2005; Epelman et al. 2007). The hallmark pathological finding of NEC is ischaemic or coagulative necrosis, which is characterised by full thickness necrosis, paucity of acute inflammatory cells (neutrophils) and a predominantly lymphocytic infiltrate (Guner et al. 2008). In our group, it was not possible to stage the disease as there were not enough serial scans performed to assess the sonographic progression. The sonographic and pathological or surgical findings were well correlated in 3 individuals that had both an abdominal ultrasound examination and surgery/necropsy. Detailed complete evaluation of the Gl tract histologically may be necessary to detect focal regions of necrosis. This may be the reason for the lack of detection of a necrotising component in the pathological examination of 2 of the cases in this series. In these 2 foals in which histopathology did not find evidence of necrosis only a few areas of PI were seen sonographically, which would have been difficult to find at post mortem without guiding the pathologist to the area of interest.

Differentiating intraluminal from intramural gas by ultrasonography may be difficult in some cases. Rotating the transducer, changing subject position and the use of higher frequency transducers with excellent resolution help in distinguishing $\mathrm{PI}$ from artefacts (pseudopneumatosis intestinalis) (Kim et al. 2005, 2007). Only subjects in which the hyperechoic echoes of gas were confidently imaged within the wall were classified as having $\mathrm{Pl}$ in this study. Statistical analysis of the correlation between sonographic and histological changes was not performed owing to the small number of cases that had both. 
This study has all the weaknesses associated with its retrospective nature, including the presence of missing data. Therefore, a prospective study assessing the maturity of the foal, the feeding practices, clinical findings, clinicopathological findings, ultrasonographic findings and the results of surgical intervention should be performed to further study this disease affecting neonatal foals. Prospective examination of tissue samples from foals with sonographically diagnosed PI is indicated to further study the association between sonographic PI and pathological necrotising Gl disease. The sonographic evaluation of the neonatal intestine should include colour Doppler assessment of intestinal blood flow, as submucosal blood flow characterisation using colour Doppler sonography correlates well with histology and outcome and allows differentiation of focal from diffuse necrosis in human neonates (Faingold etal. 2005). Normal descriptions of the sonographic appearance of the neonatal GI tract, including measurements of wall thicknesses and assessment of intestinal blood flow, also need to be obtained.

In conclusion, this is the first report of the detection of PI sonographically and the evaluation of its prognostic value in neonatal foals. PI and necrotising GI disease were associated with an adverse outcome in this case series. Prematurity, the presence of blood in the faeces, gastric reflux, abdominal distension, abnormal echogenicity of the colon and leucopenia were associated with a necrotising component of the disease. The prevention, detection and clinical needs of equine neonates with necrotising $\mathrm{Gl}$ disease deserve further attention.

\section{Conflicts of interests}

No conflicts of interest have been declared.

\section{Sources of funding}

No funding was used for this retrospective study.

\section{Acknowledgements}

The contribution of the University of Pennsylvania's New Bolton Center clinicians to the patient management and data collection is gratefully acknowledged.

\section{Manufacturers' addresses}

almmunosystems, Inc., Spring Valley, Wisconsin, USA.

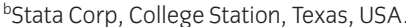

\section{References}

Babcock, D.S. (2002) Sonography of the acute abdomen in the pediatric patient. J. Ultrasound Med. 21, 887-899.

Baud, C., Saguintaah, M., Veyrac, C., Couture, A., Ferran, J.L., Barnéon, G. and Veyrac, M. (2004) Sonographic diagnosis of colitis in children. Eur Radiol. 14, 2105-2119.

Cudd, T. and Pauly, T.H. (1987) Necrotizing enterocolitis in two equine neonates. Comp. cont. Educ. Pract. 9, 88-89.

East, L.M., Savage, C.J., Traub-Dargatz, J.L., Dickinson, C.E. and Ellis, R.P. (1998) Enterocolitis associated with Clostridium perfringens infection in neonatal foals: 54 cases (1988-1997). J. Am. vet. med. Ass. 11, 1751-1756.

Epelman, M., Daneman, A., Navarro, O.M., Morag, I., Moore, A.M., Kim, J.H., Faingold, R., Taylor, G. and Gerstle, J.T. (2007) Necrotizing enterocolitis: Review of state-of the- art imaging findings with pathologic correlation. Radiographics $\mathbf{2 7}$, 285-305.

Faingold, R., Daneman, A., Tomlinson, G., Babyn, P.S., Manson, D.E., Mohanta, A., Moore, A.M., Hellmann, J., Smith, C., Gerstle, T. and Kim, J.H. (2005) Necrotizing enterocolitis: Assessment of bowel viability with color doppler US. Radiology 235, 587-594.
Guner, Y.S., Chokshi, N., Petrosyan, M., Upperman, J.S., Ford, H.R. and Grikscheit, T.C. (2008) Necrotizing enterocolitis - bench to bedside: Novel and emerging strategies. Semin. pediatr. Surg. 17, 255-265.

Gurland, B., Dolgin, S.E., Shlasko, E. and Kim, U. (1998) Pneumatosis intestinalis and portal vein gas after blunt abdominal trauma. J Pediatr. Surg. 33, 1309-1311.

Jones, R.L., Adney, W.S., Alexander, A.F., Shideler, R.K. and Traub-Dargatz, J.L. (1988) Hemorrhagic necrotizing enterocolitis associated with Clostridium difficile infection in four foals. J. Am. vet. med. Ass. 193, 76-79.

Kim, W.Y., Kim, I.O., Kim, W.S., Yeon, K.M., Kim, G.I., Lee, S.W., Suh, J.S., Choi, H.Y. and Chang, K.J. (2007) Sonographic findings in a model of ischemia-induced necrotizing enterocolitis with pathological correlations. Invest. Radiol. 42, 312-318.

Kim, W.Y., Kim, W.S., Kim, I.O., Kwon, T.H., Chang, W. and Lee, E.K. (2005) Sonographic evaluation of neonates with early-stage necrotizing enterocolitis. Pediatr. Radiol. 35, 1056-1061.

King, S. and Suckett, B. (1992) Sonographic diagnosis of portal venous gas in two pediatric liver transplant patients with benign pneumatosis intestinalis. Case reports and literature review. Pediatr. Radiol. 22, 577-578.

Lee, J.S. and Polin, R.A. (2003) Treatment and prevention of necrotizing enterocolitis. Semin. Neonatol. 8, 449-459

Levy, J.A. and Noble, V.E. (2008) Bedside ultrasound in pediatric emergency medicine. Pediatrics 121, 1404-1412.

Magdesian, K.G. (2005) Neonatal foal diarrhea. Vet. Clin. N. Am.: Equine Pract. 21, 295-312.

McAuliffe, S.B. (2004) Abdominal ultrasonography of the foal. Clin. Tech. equine Pract. 3, 308-331.

Obladen, M. (2009) Necrotizing enterocolitis - 150 years of fruitless search for the cause. Neonatology 96, 203-210.

Pastore, G.E., Lamb, C.R. and Lipscomb, V. (2007) Comparison of the results of abdominal ultrasonography and exploratory laparotomy in the dog and cat. J. Am. anim. Hosp. Ass. 43, 264-269.

Porter, M.B. and Ramirez, S. (2005) Equine neonatal thoracic and abdominal ultrasonography. Vet. Clin. N. Am.: Equine Pract. 2, 407-429.

Puylaert, J.B. (2001) Ultrasound of acute Gl tract conditions. Eur. Radiol. 11, 1867-1877

Reef, V.B. (1998) Pediatric Abdominal Ultrasonography. Equine Diagnostic Ultrasound, W.B. Saunders, Philadelphia. pp 364-405

Reef, V.B. (2004) Adult abdominal US. Clin. Tech. equine Pract. 3, 294-30.

Silva, C.T., Daneman, A., Navarro, O.M., Moore, A.M., Moineddin, R., Gerstle, J.T., Mittal, A., Brindle, M. and Epelman, M. (2007) Correlation of sonographic findings and outcome in necrotizing enterocolitis. Pediatr. Radiol. 3, 274-82.

Slovis, N.M. (2003) Gastrointestinal failure. Clin. Tech. equine Pract. 2, 79-86.

St. Peter, S.D., Abbas, M.A. and Kelly, K.A. (2003) The spectrum of pneumatosis intestinalis. Arch. Surg. 138, 68-75.

Thompson, A.M. and Bizzarro, M.J. (2008) Necrotizing enterocolitis in newborns. Pathogenesis, prevention and management. Drugs 68, 1227-1238.

Vaala, W.E. (2009) Distended and/or painful abdomen in disorders and management of the neonate. In: Large Animal Internal Medicine, 4th edn., Ed: B.P. Smith, Mosby Elsevier, St Louis. pp 306-315.

Walsh, M.C. and Kliegman, R.M. (1986) Necrotizing enterocolitis: Treatment based on staging criteria. Pediatr. Clin. North Am. 33, 179-201.

Wilkins, P.A. (2010) Disorders of foals. In: Equine Internal Medicine, 3rd edn., Eds: S.M. Reed, W.M. Bayly and D.C. Sellon, W.B. Saunders, St Louis. pp 1311-1363.

Yager, J.A. (1987) The pathogenesis of Rhodococcus equi pneumonia in foals. Vet. Microbiol. 14, 225-32.

\section{Supporting information}

Additional supporting information may be found in the online version of this article.

Video: Sonogram of the ventral abdomen of a foal with enterocolitis. Notice the hyperechoic intramural gas within a loop of thickened small intestine. The gas echoes are imaged extending from the mucosal surface into the submucosa and reach the limit between this and the muscularis. The submucosa is thickened and presents increased echogenicity.

Please note: Wiley-Blackwell are not responsible for the content or functionality of any supporting materials supplied by the authors. Any queries (other than missing material) should be directed to the corresponding author for the article. 